Review

\title{
Dynamics towards the steady state applied for the Smith-Slatkin mapping
}

\author{
Juliano A. de Oliveira ${ }^{\mathrm{a}, \mathrm{b}, *}$, Larissa C. N. Ramos ${ }^{\mathrm{b}}$, Edson D. Leonel ${ }^{\mathrm{a}}$ \\ a Universidade Estadual Paulista (UNESP), Instituto de Geociências e Ciências Exatas, Departamento de Física, Câmpus de Rio Claro, Av.24A, 1515, SP \\ 13506-900, Brazil \\ ${ }^{\mathrm{b}}$ Universidade Estadual Paulista (UNESP), Câmpus de São João da Boa Vista, Av. Profa., Isette Corrêa Fontão, 505, SP 13876-750, Brazil
}

\section{A R T I C L E I N F O}

\section{Article history:}

Received 9 October 2017

Revised 20 December 2017

Accepted 23 December 2017

Available online 2 February 2018

Keywords:

Smith-Slatkin mapping

Critical exponents

Scaling invariance

\begin{abstract}
A B S T R A C T
We derived explicit forms for the convergence to the steady state for a 1-D Smith-Slatkin mapping at and near at bifurcations. We used a phenomenological description with a set of scaling hypothesis leading to a homogeneous function giving a scaling law. The procedure is supported by numerical simulations and confirmed by a theoretical description. At the bifurcation we used an approximation transforming the difference equation into a differential one whose solution remount all scaling features. Near the bifurcation an investigation of fixed point stability leads to the decay for the stationary state. Simulations are made in the pitchfork, transcritical and period doubling bifurcations.
\end{abstract}

(C) 2018 Elsevier Ltd. All rights reserved.

\section{Introduction}

The pioneer application of nonlinear mapping for the investigation of population dynamics in biology is due to May [1]. After his publication many different contributions appeared. Applications of mappings are vast and can be seen in physics [2-6], chemistry, biology, engineering, mathematics and many others [7-17].

The investigation of stability of fixed points as well as conditions leading to bifurcations are well known [18-21]. Intermittence was investigated in Ref. [22] and led to interesting properties where a pseudo regularity along a chaotic dynamics is anticipating a tangent bifurcation giving birth to a periodic window, hence to regularity. It is known that the convergence to the fixed point at the bifurcation was proved to obey an homogeneous function characterized by a set of three critical exponents [23,24]. Near the bifurcations the dynamics converges to the steady state by means of an exponential decay [23] whose relaxation time is given by a power law for a bifurcation parameter. The set of critical exponents dictates the speed of convergence to the stationary point and can also be used to identify, whenever it is not possible analytically what type the bifurcation is. In this paper, we consider the Smith-Slatkin mapping, derived from applications in biology, and seek to obtain, understand and describe the critical exponents near the bifurcations. We implement different procedures to describe the dynamics and hence obtain the exponents. First we identify

\footnotetext{
* Corresponding author.

E-mail address: julianoantonio@sjbv.unesp.br (J.A. de Oliveira).
}

where the bifurcations are. Then we investigate the convergence to the fixed point using numerical simulations. We consider and approximation that transforms the difference equation, near the fixed point, into a differential equation, and solve it analytically to compare the arguments with the corresponding scaling times. The critical exponents emerge naturally from such a procedure and are obtained for short and large times. Near the bifurcation we obtain the relaxation time to the steady state by using fixed point stability analysis. At the bifurcation the convergence is described by an homogeneous function while near the bifurcations an exponential decay explains how the steady state is reached.

The organization of the paper is simple. Section 2 describes the mapping, the numerical simulations as well as the analytical findings. Conclusions are shown in Section 3.

\section{The model and scaling properties}

The model we consider in this paper is a version of the SmithSlatkin mapping, which is written as [25-28]

$x_{n+1}=f\left(x_{n}\right)=\frac{R x_{n}}{1+a x_{n}^{\gamma}}$

where $R, a$ and $\gamma$ are control parameters and we consider them to be non negative. The dynamical variable is represented by $x$ when the index $n$ denotes the iteration number. For the case of $\gamma=1$ the Skellam model $[28,29]$ is recovered. To give a glimpse of the orbit diagram, Fig. 1 was constructed for the parameters $\gamma=6, a=1$ for the initial condition $x_{0}=0.01$. 


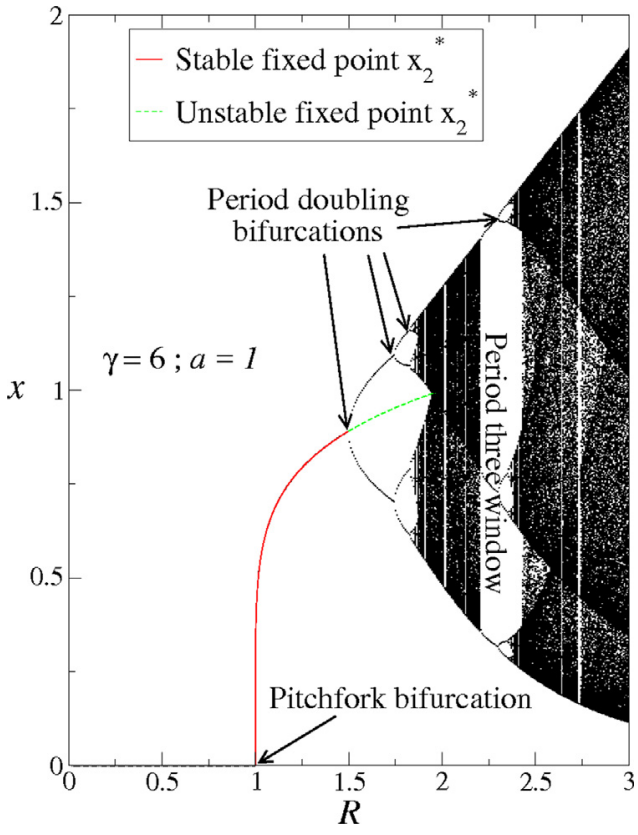

Fig. 1. Orbit diagram obtained for Eq. (1) using $\gamma=6, a=1$ and the initial condition $x_{0}=0.01$. Fixed point $x_{2}^{*}$ is represented in red (stable) and green (unstable). Bifurcations as well as the main periodic window are identified in the diagram. (For interpretation of the references to colour in this figure legend, the reader is referred to the web version of this article.)

The fixed points, obtained from the condition $x_{n+1}=x_{n}=x^{*}$ are: (i) $x_{1}^{*}=0$, which is asymptotically stable for $R \in[0,1$ ), (ii) and (iii) deserve a short discussion first. For any odd $\gamma$, fixed points are: (ii) $x_{2,3}^{*}= \pm\left[\frac{R-1}{a}\right]^{\frac{1}{\gamma}}$ while $\gamma$ is of any other kind, even, irrational etc, we obtain (iii) $x_{2}^{*}=\left[\frac{R-1}{a}\right]^{\frac{1}{\gamma}}$. Fixed points $x_{2,3}^{*}$ are asymptotically stable ${ }^{1}$ for $R \in\left(1, \frac{\gamma}{(\gamma-2)}\right)$, for $\gamma \neq 2$. A pitchfork supercritical bifurcation happens at $R=1$ for an odd $\gamma$ while a transcritical is observed at same $R$ for any other value of $\gamma$. Red curve in Fig. 1 shows the stable fixed point $x_{2}^{*}$ while green curve is a continuation of $x_{2}^{*}$ however after a period doubling bifurcation where it is unstable. A period two orbit arises at $R=\frac{\gamma}{(\gamma-2)}$ following normal Feigenbaum scaling $[30,31]$ after that.

Our first objective in this paper is to consider the convergence to the fixed point $x_{1}^{*}=0$ at the bifurcation in $R=1$. We shall show it obeys scaling properties leading different curves generated by different initial conditions to overlap onto each other, after appropriate scaling transformations, into a single and universal plot. Near the bifurcation, the convergence is so far described by an exponential decay whose relaxation time depend on the distance of the bifurcation. We use Taylor expansion near the fixed point, investigating the fixed point stability to prove it.

To illustrate how the dynamical variable evolves to the equilibrium at a bifurcation, we considered $R=1, \gamma=6, a=1$ and different initial conditions for $x_{0}$. Fig. 2(a) shows the convergence to the fixed point $x_{1}^{*}=0$. We see that for short $n$, the orbit stays confined in a regime of seemingly constant plateau. After a while eventually it suffers a changeover at a typical crossover iteration number denoted as $n_{x}$ and ultimately bends towards a regime of decay to its final state $x^{*}$.

The scaling properties extracted from Fig. 2(a) are the following: (i) For a short $n \ll n_{x}$ we notice $x(n) \propto x_{0}^{\alpha}$, leading us to con-

\footnotetext{
${ }^{1}$ By asymptotically stable we mean that given an initial condition inside of the basin of attraction of the fixed point, in the limit of $\lim n \rightarrow \infty$, the orbit has a final state at $x^{*}$, hence converging to the fixed point.
}

clude that $\alpha=1$ since $x(n) \propto x_{0}$; (ii) For large enough $n$, typically $n \gg n_{x}$, the dynamical variable is described as $x(n) \propto n^{\beta}$ where $\beta$ is a decay exponent which depends on the nonlinearity of the mapping $\gamma$. For $\gamma=6$ we obtained from fitting numerically the data an exponent $\beta=-0.16666320$ (8), as shown in the decaying regime of Fig. 2; (iii) Finally, the crossover iteration number $n_{x}$ is given by $n_{x} \propto x_{0}^{z}$ where $z$ is a changeover exponent.

A homogeneous function of the type

$x\left(x_{0}, n\right)=l x\left(l^{\tilde{a}} x_{0}, l^{\tilde{b}} n\right)$

is a natural consequence of the behavior observed from Fig. 2(a) as well as from the scaling hypotheses. Here $l$ is a scaling factor, $\tilde{a}$ and $\tilde{b}$ are characteristic exponents. Doing a similar procedure as made in Ref.[23] a scaling law appears as

$z=\frac{\alpha}{\beta}$.

The knowledge of any two exponents allows one to find the third by using Eq. (3). The relevant scaling transformations to be made are $x \rightarrow x / x_{0}^{\alpha}$ and $n \rightarrow n / x_{0}^{z}$, leading to a perfect overlap of all curves shown in Fig. 2(a) onto a single and hence universal curve, as shown in Fig. 2(b).

When the dynamical variable $x(n)$ is very close to the equilibrium, the expression $x_{n+1}=R x_{n}\left(1+a x_{n}^{\gamma}\right)^{-1}$ can be Taylor expanded leading to $x_{n+1}=R x_{n}\left(1-a x_{n}^{\gamma}\right)$. Moreover its variation as compared to the next iterate to be very small, i. e., $x_{n+1}-x_{n}$ is small enough. Such property allows us to use the following approximation $x_{n+1}-x_{n} \cong \frac{d f}{d n}$. For $R=1$, this leads to $\frac{d f}{d n}=-a x^{\gamma+1}$. This is a first order differential equations that must be solved for the ranges $x \in\left[x_{0}, x(n)\right]$ and $n$ starting from $n=0$. The solution is written as

$x(n)=\frac{x_{0}}{\left[1+a \gamma x_{0}^{\gamma} n\right]^{\frac{1}{\gamma}}}$.

Eq. (4) allows us to do the following analysis: (i) when $a \gamma x_{0}^{\gamma} n \ll 1$, we have $x(n) \cong x_{0}$, therefore leading to $\alpha=1$; (ii) For the case of $a \gamma x_{0}^{\gamma} n \gg 1$ we end up with $x(n) \approx n^{-1 / \gamma}$, hence $\beta=-1 / \gamma$; (iii) for the case $a \gamma x_{0}^{\gamma} n_{x}=1$ we have $n_{x} \propto x_{0}^{-\gamma}$, therefore $z=-\gamma$. All of these findings are giving support for the numerical simulations. Eq. (4) is plotted in Fig. 2(a) as dashed lines and we see the agreement between the numerical an analytical description is remarkable.

Near the bifurcation the dynamics is not described anymore by an homogeneous function. Instead of it the convergence is described rather by an exponential decay of the type (see Refs. $[32,33])$

$x(n)-x^{*}=\left(x_{0}-x^{*}\right) e^{-n / \tau}$,

where $\tau$ is the relaxation time described by

$\tau \propto \mu^{\delta}$

and $\delta$ is a relaxation exponent. Fig. 3 shows the behavior of $\tau v s . \mu$ given an exponent $\delta=-0.9879(4) \cong-1$, obtained by a numerical fitting of the data, and this result is invariant with respect to the parameter $\gamma$.

Let us now describe the convergence to the steady state when $R \neq 1$, therefore near the bifurcation. There is no difference on the procedure considering before or after the bifurcation. We shall consider the neighborhood of $R=R_{c}=1$, where the index $c$ denotes the critical, i.e., the bifurcation parameter. Starting from an initial condition near the fixed point we have $x_{0}=x^{*}+\epsilon_{0}$, where $x^{*}$ denotes the fixed point and $\epsilon_{0}$ corresponds to an initial distance from the fixed point. Since the mapping is given by $x_{n+1}=f\left(x_{n}\right)$, we have that $x_{1}=f\left(x^{*}+\epsilon_{0}\right)$. Since $\epsilon_{0}$ is sufficiently small, a Taylor 

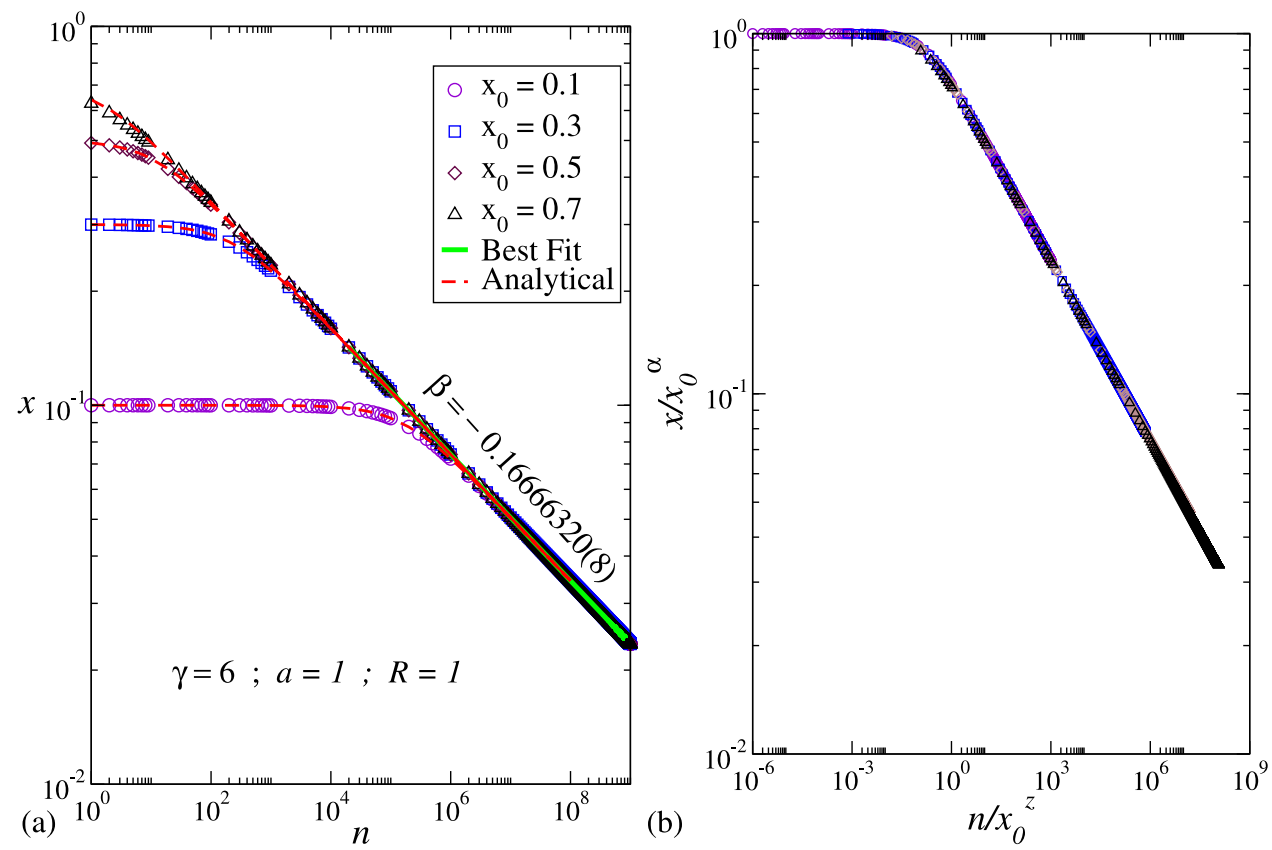

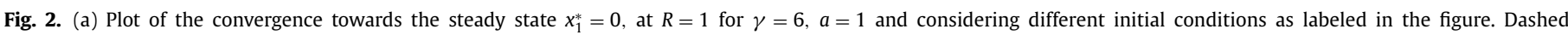

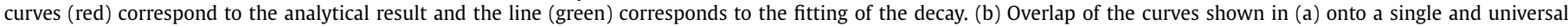

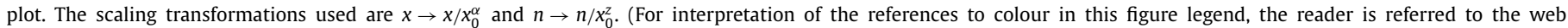
version of this article.)

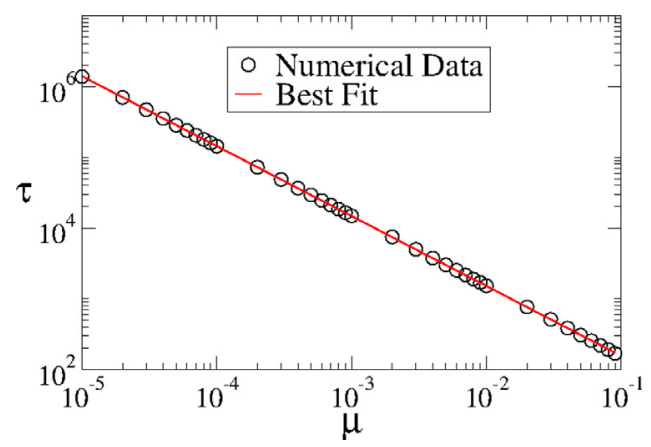

Fig. 3. Plot of $\tau v s$. $\mu$ for the control parameters $\gamma=6$ and $a=1$. A power law fitting gives $\delta=-0.9879$ (4) and correlation coefficient $c_{f}=-0.9999966$. It is invariant with respect to $\gamma$.

expansion to the expression of $f$ gives

$$
\begin{aligned}
x_{1} & =f\left(x_{0}\right) \\
& =f\left(x^{*}\right)+\left.\epsilon_{0} \frac{\partial f}{\partial x}\right|_{x^{*}}+\left.\frac{\varepsilon_{0}^{2}}{2} \frac{\partial^{2} f}{\partial x^{2}}\right|_{x^{*}}+\ldots
\end{aligned}
$$

Keeping only first order and from the fixed point condition $f\left(x^{*}\right)=$ $x^{*}$ we have $x_{1}-x^{*}=\epsilon_{1}=\left.\epsilon_{0} \frac{\partial f}{\partial x}\right|_{x^{*}}$. When going further to the second iterated of the mapping we obtain $\epsilon_{2}=\left.\epsilon_{1} \frac{\partial f}{\partial x}\right|_{x^{*}}$ that may be rewritten in a convenient way as $\epsilon_{2}=\epsilon_{0}\left(\left.\frac{\partial f}{\partial x}\right|_{x^{*}}\right)^{2}$. Generalizing for the iterated $n$ we have

$\epsilon_{n}=\epsilon_{0}\left(\left.\frac{\partial f}{\partial x}\right|_{x^{*}}\right)^{n}$.

The condition $-1<\left.\frac{\partial f}{\partial x}\right|_{x^{*}}<1$ warrants convergence to the stationary state. Let us now consider a specific case. Fixed point $x_{1}^{*}=0$ and $R<1$ but close to one with $R_{c}=1$. The distance from the fixed point at iterated $n$ can be written in a convenient way as

$$
\left.\epsilon_{n}=\epsilon_{0} e^{\ln \left[\frac{\partial f}{\partial x} \mid\right.}\right]_{x^{*}}^{n},
$$

where $\epsilon_{n}=x_{n}-x^{*}$. Since for $x_{1}^{*}=0$ we have $\left.\frac{\partial f}{\partial x}\right|_{x^{*}}=R$, then $\epsilon_{n}=$ $\epsilon_{0} e^{n \ln (R)}$. Let us now write $R=R_{c}-\mu$ where $\mu$ gives the distance from the bifurcation. $R_{C}=1$ identifies the bifurcation parameter. Substituting the expression of $R$ in $\ln (R)$ we have

$$
\begin{aligned}
\ln (R) & =\ln \left(R_{c}-\mu\right), \\
& =\ln \left[R_{c}\left(1-\frac{\mu}{R_{c}}\right)\right], \\
& =\ln \left(R_{c}\right)-\frac{\mu}{R_{c}},
\end{aligned}
$$

where the last expression comes from Taylor expansion. The leading term is then written as

$\epsilon_{n}=\epsilon_{0} e^{-\mu n}$.

Because the term $\epsilon_{n}=x_{n}-x^{*}$ and $\epsilon_{0}=x_{0}-x^{*}$ an immediate comparison with Eq. (5) can be made. We conclude that $\delta=-1$, in well agreement with Fig. 3.

The procedure made for $R=1$ can also be made at the period doubling bifurcation as well no matter if first, second or any other order of the period doubling bifurcation. For the first period doubling, the mapping must be iterated twice. For the second period doubling bifurcation, the mapping must iterated four times and so on. Our results for the case of $R=\frac{\gamma}{\gamma-2}$, i.e., at the first period doubling bifurcation gives $\alpha=1, \beta=-1 / 2, z=-2$ and $\delta=-1$.

\section{Conclusions}

As a short summary we have investigated the convergence to the steady state in a 1-D Smith-Slatkin mapping near three bifurcations: (i) a pitchfork for $\gamma$ odd; (ii) transcritical for any other $\gamma$, which leads to similar results as of case (i) and; (iii) period 
doubling bifurcation, no matter the order considered for the bifurcation. At the bifurcation the decay is described by homogeneous function leading to three critical exponents $\alpha=1, \beta=-1 / \gamma$ and $z=-\gamma$. The relevant scaling law describing the invariance is $z=$ $\alpha / \beta$. Near the bifurcation the convergence to the fixed point is given by an exponential decay and the relaxation time is described by a power law of the type $\tau \propto \mu^{\delta}$, with $\delta=-1$ independent of the three bifurcations considered. Such exponent was obtained via fixed point investigation.

The procedure discussed here is an extension of the results presented in Ref. [23]. Generalizations for other mappings, particularly for the case of 2-D can be made. For such type of mappings, the convergence is observed in a plane. One has to find convenient variables, possible the distance from the fixed point - polar coordinates is a natural candidate since the convergence spirals around the fixed point - and describe in such coordinates the evolution towards the steady state. With this set of variables the scaling should emerge easily.

\section{Acknowledgments}

JAO thanks CNPq (421254/2016-5) (311105/2015-7) and FAPESP(2014/18672-8). LCNR thanks PROPe/UNESP and FAPESP (2017/17294-8). EDL acknowledges support from CNPq (303707/2015-1), FAPESP (2017/14414-2) and FUNDUNESP.

\section{References}

[1] May RM. Science 1974;86:645.

[2] Grebogi C, Ott E, Yorke JA. Phys Rev Lett 1982;48:1507. Physica D 7 (1983), 181.
[3] Pounder JR, Rogers TD. Nonlinear Anal Theor Methods Appl 1986;10:415.

[4] Joglekar M, Ott E, Yorke A. Phys Rev Lett 2014;113. 084101.

[5] Gallas JAC. Phys Rev Lett 1983;70:2714.

[6] Collet P, Eckmann J-P. Iterated maps on the intervalas dynamical systems. Boston, MA: Birkhauser; 1980.

[7] May RM, Oster GA. Am Nat 1976;110:573.

[8] Hamacher K. Chaos 2012;22:033149.

[9] McCartney M. Chaos 2012;21:043104

[10] Philominathan P, Santhiah M, Mohamed IR, et al. Int J Bifurcations Chaos 2011;21:1927.

[11] Santhiah M, Philominathan P. Pramana J Phys 2010;75:403.

[12] Zhang Y-G, Zhang J-F, Ma Q, et al. Int J Nonlinear Sci Numer Simul 2010;11:157.

[13] Wen H, Guang-Hao Z, Gong Z, et al. Acta Physica Sinica 2012;17:170505

[14] Urquizu M, Correig AM. Chaos, Solitons Fractals 2007;33:1292.

[15] Livadiotis G. Adv Complex Syst 2005;8:15.

[16] Ilhem D, Amel K. Discrete Dyn Nat Soc 2006;2006. Article ID 15840.

[17] Li TY, Yorke JA. Am Math Monthly 1975;82:985.

[18] Martelli M. Introduction to discrete dynamical systems and chaos. New York: Wiley; 1999 .

[19] Devaney RL. A first course in chaotic dynamical systems: Theory and experiment (studies in nonlinearity). Westview Press; 1992.

[20] Galor O. Discrete dynamical systems. Heildelberg: Springer; 2007.

[21] Devaney RL. An introduction to chaotic dynamical systems. Cambridge: Westview Press; 2003.

[22] Hirsch JE, Huberman BA, Scalapino DJ. Phys Rev A 1982;25:519.

[23] Teixeira RMN, Rando DS, Geraldo FC, Filho RNC, de Oliveira JA, Leonel ED. Phys Lett A 2015;379:1246.

[24] Leonel ED, Teixeira RMN, Rando DS, Filho RNC, de Oliveira JA. Phys Lett A 2015;379:1796.

[25] Maynard Smith J, Slatkin M. Ecology 1973;54:384.

[26] Stone L, Hart D. Theor Popul Biol 1999;55:227.

[27] Doebeli M, Jong G. Theor Popul Biol 1999;55:37.

[28] Nedorezov LV, Sadykova D. Ecol Modell 2008;212:162.

[29] Skellam JG. Biometrica 1951;38:196.

[30] Feigenbaum MJ. J Stat Phys 1979;21:669.

[31] Feigenbaum MJ. J Stat Phys 1978;19:25.

[32] Leonel ED, da Silva JKL, Kamphorst SO. Int J Bifurcation Chaos 2002;12:1667.

[33] Hohenberg PC, Halperin BI. Rev Mod Phys 1977;49:435. 